\title{
ERRATUM
}

\section{Superpolynomial Speedups Based on Almost Any Quantum Circuit}

\author{
Sean Hallgren ${ }^{1}$ and Aram W. Harrow ${ }^{2}$ \\ ${ }^{1}$ Department of Computer Science and Engineering, The Pennsylvania State University \\ University Park, PA \\ ${ }^{2}$ Department of Mathematics, University of Bristol, Bristol, U.K. \\ a.harrow@bris.ac.uk
}

L. Aceto et al. (Eds.): ICALP 2008, Part I, LNCS 5125, pp. 782-795, 2008.

(C) Springer-Verlag Berlin Heidelberg 2008

DOI 10.1007/978-3-540-70575-8_73

The information that the articles were retrieved from arXiv.org is missing in the following references:

\section{References}

[Aar03] Aaronson, S.: Quantum lower bound for recursive Fourier sampling. Quantum Information and Computation 3(2), 165-174 (2003), arXiv:quant$\mathrm{ph} / 0209060$

[AJL06] Aharonov, D., Jones, V., Landau, Z.: A polynomial quantum algorithm for approximating the jones polynomial. In: STOC 2006: Proceedings of the thirty-eighth annual ACM symposium on Theory of computing, pp. 427-436. ACM Press, New York (2006), arXiv:quant-ph/0511096

[BCvD05] Bacon, D., Childs, A.M., van Dam, W.: From optimal measurement to efficient quantum algorithms for the hidden subgroup problem over semidirect product groups. In: FOCS 2005: 46th Annual IEEE Symposium on Foundations of Computer Science, pp. 469-478 (2005), arXiv:quant-ph/0504083

[DOP07] Dahlsten, O.C.O., Oliveira, R., Plenio, M.B.: Emergence of typical entanglement in two-party random processes. J. Phys. A 40, 8081-8108 (2007), arXiv:quant-ph/0701125

[FvdG99] Fuchs, C.A., van de Graaf, J.: Cryptographic distinguishability measures for quantum mechanical states. IEEE Trans. Inf. Th. 45(4), 1216-1227 (1999), arXiv:quant-ph/9712042

[HH08] Hallgren, S., Harrow, A.W.: Superpolynomial speedups based on almost any quantum circuit (2008), arXiv:0805.0007 
$\left[\mathrm{HMR}^{+}\right.$06] Hallgren, S., Moore, C., Rötteler, M., Russell, A., Sen, P.: Limitations of quantum coset states for graph isomorphism. In: STOC 2006: Proceedings of the 38th Annual ACM Symposium on Theory of Computing, pp. 604617. ACM Press, New York (2006), arXiv:quant-ph/0511148, arXiv:quant$\mathrm{ph} / 0612089$

[PSW06] Popescu, S., Short, A.J., Winter, A.: The foundations of statistical mechanics from entanglement: Individual states vs. averages. Nature 2, 754-758 (2006), arXiv:quant-ph/0511225

[Sho97] Shor, P.W.: Polynomial-time algorithms for prime factorization and discrete logarithms on a quantum computer. Siam J. Comp. 26(5), 1484-1509 (1997), arXiv:quant-ph/9508027

[Wat01] Watrous, J.: Quantum algorithms for solvable groups. In: STOC 2001: Proceedings of the Thirty-Third Annual ACM Symposium on Theory of Computing, Crete, Greece, pp. 60-67. ACM Press, New York (2001), arXiv:quant-ph/0011023 